\title{
A MENSURAÇÃO DA IMPORTÂNCIA DE ATRIBUTOS EM SERVIÇOS: UMA COMPARAÇÃO DE ESCALAS
}

THE MEASUREMENT OF THE SERVICE ATTRIBUTES IMPORTANCE: A SCALE COMPARISON

MARCOS ANGELIGHISI

Mestrando em Administração na FEARP/USP.

Av. Bandeirantes, 3.900 - Ribeirão Preto - SP - CEP 14040-900

E-mail:mghisi@bol.com.br

EDGARD MONFORTE MERLO Doutor em Administração pela FEA/USP. Professor na FEARP/USP. Av. Bandeirantes, 3.900 - Ribeirão Preto - SP - CEP 14040-900

E-mail edgardmm@usp.br

MARCELO SEIDO NAGANO Pós-doutorado pela LAC/INPE. Professor na EESC/USP. Av. Trabalhador São-carlense, 400 - São Carlos - SP - CEP 13560-970 


\section{RESUMO}

Este artigo discute especificamente aspectos metodológicos da mensuração de atributos em serviços para um contexto de pesquisa quantitativa. Neste caso, uma abordagem de questionário auto-aplicado foi utilizada. O artigo inicia descrevendo alguns aspectos da decisão de marketing em relação ao uso de informações. A seguir, algumas questões metodológicas, relacionadas a escalas, foram analisadas e discutidas com relação à pesquisa de marketing. O estudo propõe o desenvolvimento da abordagem de pontos decrescentes para a mensuração da importância de atributos. Um estudo exploratório foi conduzido para comparar os resultados da utilização da escala de diferencial semântico, a abordagem de Carvalho e Leite (I999) e dois formatos da abordagem de pontos decrescentes para avaliar os principais atributos que influenciariam os consumidores na escolha de um salão de beleza. Os resultados mostraram que a abordagem de Carvalho e Leite conduziu a resultados mais próximos da realidade e com maior poder discriminatório entre as três escalas analisadas.

\section{PALAVRAS-CHAVE}

Serviços; Importância de atributos; Escalas de mensuração; Gestão de serviços; Qualidade em serviços.

\section{ABSTRACT}

This article discusses specifically the methodological aspects of measurement of attributes in services for a context of quantitative research. In this case a self-applied questionnaire approach was used. The article begins describing some aspects of marketing decisions and the relationship with the information. Some measurement methodological aspects, mainly scales, in quantitative research was analyzed and discussed. The study proposes the decreasing points approach for the measurement of the attributes importance. An exploratory study was made to compare the scale utilization results of semantic differential, the scale of Carvalho and Leite (I999) and two approach formats of decreasing points to evaluate the main attributes that would influence consumers in the 
choice of a beauty parlor. Results pointed that Carvalho and Leite approach was the more realistic and discriminatory comparing with the three analyzed scales.

\section{KEYWORDS}

Services; Attributes importance; Measurement scales; Services management; Services quality.

\section{INTRODUÇÃO}

As decisões de marketing envolvem diversas atividades, desde estudos sobre o potencial e a participação do mercado até a avaliação da satisfação do cliente e seu comportamento de compra, como também questões relacionadas ao apreçamento, produto, distribuição e promoção (KOTLER, I999).

A maioria das pesquisas de marketing realizada é de pesquisa conclusiva descritiva (MATTAR, I994). Como destaca Churchill (I99I), a pesquisa descritiva é utilizada quando o propósito do pesquisador é: I. descrever as características de certos grupos; 2. estimar a proporção de elementos numa população específica que se comportam de uma certa forma; e 3. fazer predições específicas, isto é, descobrir ou verificar a existência de relação entre variáveis.

Este artigo trata especificamente da questão metodológica da mensuração da importância de atributos em serviços para um contexto de pesquisa quantitativa, em que a abordagem de comunicação de questionário auto-aplicado é utilizada. A discussão a seguir apresenta algumas utilidades que essa informação possui para os fornecedores de serviço. Na seção 3, são discutidas algumas considerações teóricas a respeito das escalas utilizadas em marketing que podem ser utilizadas para esta finalidade para então, na seção 4, apresentar um novo procedimento de mensuração de atitudes. Os resultados e as considerações finais do teste empírico realizado com a abordagem de pontos decrescentes são apresentados, respectivamente, nas seções 5 e 6 .

\section{POR QUE MENSURAR A IMPORTÂNCIA DE ATRIBUTOS DE SERVIÇOS?}

Do ponto de vista dos prestadores de serviço, a finalidade da mensuração da importância que os atributos ou processos de serviço possuem para os clientes pode representar uma fonte potencial de informação para ser utilizada, sobretudo, de três maneiras principais: a) na segmentação dos clientes e ajuste da oferta de serviços das empresas; b) no controle das interações de serviço, identificando os 
atributos críticos de serviço para então estabelecer padrões satisfatórios de entrega de serviço; e c) na mensuração da qualidade e satisfação de serviço percebido.

Os mercados consistem fundamentalmente de compradores que diferem entre si em um ou mais aspectos. Podem diferir em termos de desejos, poder de compra, localização geográfica, atitudes e práticas de compra. Uma poderosa forma de segmentação é classificar os compradores de acordo com os diferentes benefícios que buscam em um produto (KOTLER, I999).

Benefícios constituem, segundo Weinstein (I995), o conjunto das vantagens ou satisfações que um produto proporciona às necessidades ou desejos dos consumidores, indo além das características de produto, e servem para satisfazer necessidades físicas, emocionais ou psicológicas.

O conceito de saliência referenciado por Engel et al. (2000) na literatura de comportamento de compra do consumidor pode ser também ampliado para sua aplicação no setor de serviços. Segundo a definição dos autores, o conceito de saliência reflete a noção de que os critérios de avaliação geralmente diferem na sua influência sobre as seleções de produto dos consumidores nas circunstâncias em que alguns critérios acabam tendo maior impacto do que outros na seleção de produtos. Generalizando para a área de serviços, alguns atributos de serviço são mais importantes do que outros e, desta forma, possuem maior impacto na percepção de valor geral de um serviço.

Um benefício principal ou um conjunto de benefícios é freqüentemente construído e utilizado para propósitos de identificação de segmentos. O uso prudente da análise de segmentação por benefícios fornece aos profissionais de marketing uma nova perspectiva e adiciona um insight às situações de mercado. Quando executada com propriedade, essa abordagem é uma das mais poderosas maneiras de identificar e explorar mercados (WEINSTEIN, I995). Assim, os prestadores de serviço podem utilizar esta informação de valor dos benefícios buscados para ajustar sua oferta, diversificando seus serviços de acordo com as diferentes necessidades de seus clientes. Sabendo-se disso, o prestador de serviço poderia diferenciar a sua oferta de serviços, cobrando mais caro de clientes que necessitem rapidamente do conserto de seus veículos.

A respeito desse fato, é interessante lembrar o conceito do excedente do consumidor, que, segundo Pindyck e Rubinfeld (2002), pode ser compreendido como o benefício líquido obtido pelo consumidor por ser capaz de comprar um bem ao preço vigente no mercado. Segmentar os clientes pelos benefícios que eles procuram na prestação de serviços envolve, assim, a identificação dos atributos de serviços que os clientes consideram como mais importantes para eles. Assim pode-se perceber que a metodologia quantitativa de pesquisa pode ser uma ferramenta de grande valia para a mensuração deste conceito. 


\section{CONTROLE DAS INTERAÇÕES DE SERVIÇOS}

Como aborda Gianesi e Corrêa (I994), a percepção do cliente em relação ao serviço é formada em cada uma das interações que o cliente tem com a empresa prestadora de serviço. Talvez a melhor expressão para descrever este processo de interação seja a expressão "momentos da verdade", criada por Normann (I994 apud GIANESI; CORRÊA, I994) para simbolizar o momento de contato entre o fornecedor do serviço e o cliente. A prestação de serviços envolve a vivência, por parte do cliente, de uma série de momentos da verdade; a percepção do cliente em relação ao serviço é formada em cada uma dessas interações.

Porém, nem todos os momentos da verdade têm a mesma importância para o cliente e há certos momentos da verdade críticos ou fundamentais para a percepção do cliente a respeito do serviço prestado. Isso leva o fornecedor de serviços a procurar identificar esses momentos da verdade críticos, para que possa priorizar seus esforços visando gerar uma percepção favorável do serviço para o cliente (GIANESI; CORRÊA, I994).

Zeithaml e Bitner (2003) lembram que compreender as expectativas e percepções dos clientes é o primeiro passo para a prestação de um serviço de qualidade.

Neste momento, é interessante ressaltar o conceito de zona de tolerância descrito por Lovelock e Wright (200I) como o grau em que os clientes estão dispostos a aceitar a variação na entrega de serviço.

\section{MENSURAÇÃO DA QUALIDADE E SATISFAÇÃO EMI SERVIIÇOS}

As pesquisas realizadas na área de qualidade em serviços pressupõem muitas vezes que os clientes possuem expectativas e que elas representam um certo padrão ou ponto de referência usado pelos clientes para avaliar a performance de uma empresa de serviços (ROBLEDO, 200I). Contudo, a inclusão ou não das expectativas como determinante da qualidade de serviço percebida tem resultado em dois paradigmas conflitantes. Um primeiro seria o paradigma da desconfirmação: nesta abordagem, os clientes avaliam um serviço comparando suas percepções do serviço recebido com suas expectativas iniciais (PARASURAMAN et al., I985, I988, I994; BROWN et al., I993). O segundo seria o paradigma da percepção, cujos defensores afirmam que as expectativas são irrelevantes e até mesmo corrompem a informação de um modelo planejado para avaliar a qualidade de serviço percebida. Eles afirmam que as percepções dos clientes são as únicas medidas necessárias (CRONIN; TAYLOR, I992). 
A complexidade da discussão anterior aumenta quando modelos ponderados ou não ponderados pela importância dos atributos, do ponto de vista do cliente, são utilizados na mensuração da qualidade de serviços. Entretanto, muitos pesquisadores da área de serviços discordam do uso de modelos ponderados, talvez pela dificuldade ou, mais propriamente, do uso inadequado de métodos de mensuração da importância dos atributos que, segundo eles, não acrescentam poder de predição aos modelos.

Na versão inicial do instrumento Servqual, a qualidade de serviço era mensurada simplesmente pelas medidas de expectativas e performances, não havendo medições de importância dos atributos (PARASURAMAN; ZEITHAML; BERRY, I988). Porém, conforme Carman (I990), devido ao fato de a importância de cada atributo ser bastante distinta das expectativas dos clientes, a medição da importância dos atributos é relevante para a qualidade de serviços. A partir disso, Parasuraman, Berry e Zeithaml (I99I) introduziram a ponderação por importância de atributos na escala Servqual. Nesse refinamento da escala, os respondentes eram solicitados a alocar too pontos em itens que descreviam as cinco dimensões da qualidade de serviços. Essas ponderações eram então utilizadas para fornecer uma ponderação média para a qualidade geral de serviços.

Em estudo realizado por Cronin e Taylor (I992), foi testado o uso da ponderação por importância em conjunto com duas escalas, Servqual e Servperf, adotando uma abordagem diferente da de Parasuraman, Berry e Zeithaml (I99I). Nessa abordagem, os respondentes eram solicitados a determinar os escores de importância, em uma escala variando de um a sete, para cada um dos 22 atributos mensurados. No teste empírico realizado, os autores concluíram que o uso da ponderação não melhorou em nada o poder preditivo dos instrumentos, mas de fato o reduziu. Situação similar a essa foi a de Teas (I993), que também conclui que as escalas ponderadas produziram efeitos piores do que as versões não ponderadas. Porém, em teste empírico realizado por Quester, Wilkinson e Romaniuk (I995) utilizando a mesma metodologia usada por Cronin e Taylor (I992) para mensuração da importância dos atributos, os autores chegaram a conclusões opostas. A adição da ponderação por importância dos atributos melhorou o poder preditivo de ambas as escalas, embora de maneira não significante.

Em resposta às considerações de Cronin e Taylor (1992), Parasuraman, Zeithaml e Berry (I994) argumentaram contra a ponderação individual de atributos. Eles acreditam que o uso de escores individuais de importância como variáveis independentes na análise de regressão é uma forma de dupla contagem, visto que o propósito primário da análise de regressão é derivar os pesos de importância por meio dos coeficientes beta. Em réplica, Cronin e Taylor (I994) consideram o oposto verdadeiro, acreditando que a derivação matemática é bas- 
tante distinta das percepções de importância expressadas pelos respondentes quando perguntadas diretamente.

Taylor (I995), utilizando evidência da literatura, sugeriu que perguntar explicitamente pela importância dos atributos pode ser um procedimento não confiável. O autor referenciado, ele sugere que sejam utilizados métodos indiretos, nos quais a ponderação de importância seria derivada estatisticamente.

Como revela Aaker, Kumar e Day (200I), a mensuração pode ser definida como o processo padronizado de atribuição de números ou outros símbolos a certas características de objetos de interesse, de acordo com algumas regras préespecificadas. Malhotra (200I) destaca que o aspecto mais importante da medição é a especificação de regras para atribuir números às características, as quais devem ser padronizadas e aplicadas uniformemente.

Uma escala é um processo de criação de um continuum, no qual os objetos são identificados de acordo com a quantidade que possuem da característica medida. Para realizar essas medições, Kinnear e Taylor (I985) argumentam que o pesquisador precisa desenvolver instrumentos adequados para que as medidas efetuadas correspondam efetivamente ao que se deseja medir (validade) e para que o erro amostral seja o menor possível (confiabilidade) diante dos recursos disponíveis. Assim, seria interessante abordar algumas questões metodológicas pertinentes relacionadas aos erros de mensuração.

Pereira (200I) ressaltou que três fatores podem induzir a erro de mensuração, a saber: I. o objeto: não só suas características intrínsecas como suas possíveis variações naturais; 2. o instrumento de medida: não só sua adequação, mas suas variações de precisão; e 3. o observador: não só suas limitações físicas, mas as variações de juízo que faça de suas observações.

Pasquali (I997) destacou que, independentemente da qualidade dos itens, a resposta aos mesmos pode ser desvirtuada por fatores relativos ao sujeito que a eles reage. Esses vieses na resposta falseiam os dados, introduzindo correlações espúrias, mesmo em se tratando de bons instrumentos. Segundo o autor, esses erros podem ser classificados em três categorias em termos de suas causas: I. cultura, relacionada ao problema de transferência de instrumentos psicológicos para outras populações para as quais eles não foram especificamente construídos e validados; 2. a resposta ao acaso, ocasionada por fatores aleatórios e não sistemáticos, como, por exemplo, a má disposição do sujeito em responder ao teste, a incompreensão das instruções e outros; e 3. a resposta estereotipada, isto é, respostas tendenciosas devido a peculiaridades do sujeito que responde, ocorre com freqüência em testes de personalidade e de atitude.

Em relação à resposta estereotipada, Pasquali (I997) destacou duas causas possíveis da ocorrência desse tipo de erro: a desejabilidade social, representando um traço de personalidade que afeta negativamente a objetividade nas res- 
postas de auto-relato e a resposta sistemática, que ao contrário da anterior, é constituída por erros de julgamento.

Especificamente, o controle dos erros de resposta sistemática tem se mostrado ainda bastante falho na utilização de escalas de avaliação (PASQUALI, I997). Ainda segundo o autor, existem vários tipos de erros de resposta sistemática: o efeito halo, o erro de leniência, a tendência central e o erro de contraste.

O efeito halo ocorre quando "um avaliador tende a avaliar um indivíduo de modo semelhante em todas as dimensões" (GUILFORD, I959, apud PASQUALI, I997). Wirtz (200I) descreve duas formas de efeito halo. Primeiramente, a resposta para um atributo em particular pode ser influenciada pela impressão geral do objeto como um todo ou seus traços afetivos. Em segundo lugar, a resposta para outros atributos pode ser influenciada pela avaliação de um atributo dominante.

O erro de leniência consiste em dizer "apenas coisas boas a respeito de todo mundo” (DUNNETTE, I983, apud PASQUALI, I997). Estatisticamente, essa tendência é definida como "uma mudança significativa na média das avaliações na direção favorável, de uma condição de avaliação para outra" (SHARON; BARTLETT, I969 apud PASQUALI, I997).

A tendência central ocorre quando um avaliador tende a colocar todos os sujeitos no centro da escala, evitando proferir julgamentos extremos.

O erro de contraste consiste na tendência de as pessoas avaliarem os outros de modo oposto ao que avaliam a si mesmas. Finalmente, Pasquali (I997) ressalta que os pesquisadores têm procurado maneiras de contornar os erros sistemáticos, como, por exemplo, eliminar o ponto neutro (central) da escala para inviabilizar a tendência central ou eliminar a parte inferior da escala para descaracterizar a leniência. Entretanto, tais investidas não têm surtido efeitos suficientes e claros.

Em relação ao método de coleta de dados, os questionários auto-aplicados possuem vantagens e desvantagens. Aaker, Kumar e Day (200I) citam que uma das vantagens das enquetes pelo correio é a de que trazem resultados mais acurados entre aqueles que respondem a elas, uma vez que o questionário é respondido à vontade pelo respondente e, assim, as respostas tendem a ser mais bem pensadas e outras pessoas podem ser consultadas para informações adicionais. Como desvantagens, os autores citam a possibilidade de que um grande número de variáveis seja controlado inadequadamente, afetado principalmente pela ordem na qual as perguntas são colocadas e o entendimento que o respondente faz das perguntas.

Conforme Malhotra (200I), as técnicas de escalonamento comumente empregadas em pesquisa de marketing podem ser classificadas em escalas comparativas e escalas não comparativas. As escalas comparativas envolvem a compa- 
ração direta de objetos e incluem o escalonamento por comparação por pares, o escalonamento por ordem de posto, o escalonamento de soma constante e o tipo Q. Nas escalas não comparativas, cada objeto é escalonado independentemente dos outros no conjunto de estímulo, admitindo-se que os dados resultantes sejam escalonados por intervalo ou por razão. A seguir, é apresentada uma breve descrição de cada uma dessas técnicas.

\section{ESCALONAMENTO POR COMPARAÇÃO POR PARES}

Como evidencia Malhotra (200I), nesta técnica apresentam-se aos entrevistados dois objetos para que escolham um entre eles, de acordo com algum critério. A técnica requer que todos os emparelhamentos possíveis entre os objetos, tomados dois a dois, sejam realizados, isto é, para $n$ objetos analisados, deverão ser realizadas $n(n-\mathrm{I}) / 2$ comparações. Uma forma de analisar os dados é somar, para todos os respondentes, o número de vezes em que um objeto é preterido por outro e então dividir esta soma pelo número de respondentes, multiplicando o resultado por Ioo. Algumas desvantagens dessa escala são a possibilidade de a ordem de apresentação tornar os resultados tendenciosos e a ocorrência de violações da suposição da transitividade de preferência.

\section{ESCALONAMENTO POR ORDEM DE POSTO}

Neste tipo de escalonamento, são apresentados simultaneamente vários objetos aos entrevistados, os quais devem ordená-los ou atribuir-lhes postos de acordo com algum critério. Também chamada de escala de ranqueamento forçada (COOPER; SCHINDLER, 2003), esta técnica força o entrevistado a discriminar entre os objetos de estímulo. Em relação à técnica emparelhada, a técnica de escalonamento por ordem de posto, segundo Malhotra (200I), toma menos tempo do entrevistado em virtude de que $n$-I decisões devem ser tomadas, em contraposto à $n(n-\mathrm{I}) / 2$ decisões da técnica emparelhada. Além disso, o autor destaca como vantagem o fato de que esta técnica elimina respostas intransitivas e suas instruções são de fácil entendimento para os entrevistados. Sendo uma técnica comparativa por natureza, sua principal desvantagem, como era de se esperar, é que gera apenas dados ordinais. Esta técnica pode ser utilizada, no contexto desta pesquisa, para pedir aos respondentes que classifiquem, por ordem de importância, uma lista de atributos de serviços.

\section{4 - 3 ESCALONAMENTO DE SOMA CONSTANTE}

Nesta técnica, os entrevistados atribuem uma soma constante de unidades, como, por exemplo, pontos, dentro de um conjunto de objetos de estímulo, ba- 
seados em algum critério predefinido. Os atributos são escalonados contandose os pontos atribuídos a cada um deles por todos os participantes e dividindose pelo número de respondentes. No contexto desta pesquisa, esta técnica pôde ser utilizada para pedir aos respondentes que distribuam ioo pontos a uma lista de atributos de serviços, de uma forma que reflita a importância que eles dão a cada atributo. Isto é, serão considerados mais importantes aqueles atributos que receberem mais pontos dos respondentes.

\subsection{ESCALONAMENTO TIPOQ}

Segundo Malhotra (200I), esta técnica tem por objetivo estabelecer uma discriminação rápida entre um número relativamente grande de objetos utilizando um processo de ordenação por postos em que os objetos são separados em pilhas com base em algum critério. O número de objetos a serem empilhados não deve ser menor do que 60 nem maior do que I49, sendo 60 a 90 objetos o alcance razoável. Entretanto, Mattar (1994) sugere que esta técnica é geralmente utilizada para a ordenação de 9 a 15 objetos e quando sua ordenação por outros métodos causaria fadiga e/ou confusão aos respondentes. Segundo o autor, o método consiste em fazer com que a ordenação seja feita passo a passo para facilitar o julgamento do respondente. Por fim, por causa da dificuldade de sua aplicação na prática, a técnica de ordenação tipo Q é muito pouco utilizada em marketing.

\section{4 - 5 ESCALA LIKERT}

Amplamente utilizada em pesquisa de marketing, a escala Likert exige que os entrevistados indiquem o grau de concordância ou discordância com cada uma de uma série de afirmações sobre objetos de estímulo. A análise pode ser feita item por item, analisando o perfil, ou então calculando um escore total para cada respondente somando-se todos os itens (MALHOTRA, 200I). Como lembra Aaker, Kumar e Day (200I), uma importante premissa desse método é que cada um dos itens (afirmações) meça algum aspecto de um mesmo fator comum. Caso contrário, os itens não poderão ser somados. Assim, a escala resultante é unidimensional.

Segundo Malhotra (200I), a escala Likert possui diversas vantagens, entre elas a facilidade de construção e aplicação, como também o entendimento por parte dos entrevistados. Como desvantagem, o autor destaca que a escala Likert exige maior tempo para ser completada do que outras escalas de classificação por itens, visto que os respondentes precisam ler cada uma das afirmações. Para sua utilização na mensuração de importância de atributos de serviço, é necessá- 
rio redigir frases enfatizando a importância (positivo) ou não (negativo) para cada um dos atributos. Dessa forma, os respondentes assinalariam a sua concordância ou não com as frases.

\section{6 ESCALA DE DIFERENCIAL SEMÂNTICO}

Trata-se de uma escala de classificação de sete pontos, em que os pontos extremos estão associados a rótulos bipolares (adjetivos) e os participantes assinalam o espaço em branco que melhor indica como descreveriam os objetos que estão sendo avaliados (MALHOTRA, 200I). De forma semelhante, Aaker, Kumar e Day (200I) descrevem a escala de diferencial semântico como a avaliação de atitudes-objetos, por parte dos respondentes, em uma série de escalas de cinco ou sete pontos, limitadas em cada ponta por frases ou adjetivos polares. Segundo os autores, o respondente escolhe um ponto cujo adjetivo mais se aproxime da descrição daquele objeto. Malhotra (200I) destacou como ponto forte da técnica a versatilidade da escala diferencial semântica e, como aspecto negativo, revelou que existe certa controvérsia sobre se os dados obtidos devem ser tratados como uma escala intervalar, o que limitaria o uso de estatísticas para sua análise. Entretanto, Kinnear e Taylor (1985) argumentaram que se forem atribuídos valores numéricos aos itens da escala, e os mesmos exibidos aos respondentes, é possível assumir que os julgamentos dos respondentes foram efetuados segundo uma escala de intervalo, e assim, os dados podem ser analisados segundo as propriedades desta escala. Porém, os autores também citam que esse procedimento tem gerado muita controvérsia entre os estudiosos de escalas.

\section{7 ESCALA STAPEL}

Segundo Malhotra (200I), a escala de Stapel é uma escala de classificação unipolar com dez categorias numeradas de $-5 \mathrm{a}+5$, sem ponto neutro. Os respondentes devem indicar o grau de precisão ou de imprecisão com que cada termo descreve o objeto, selecionando uma categoria de resposta numérica apropriada. Como coloca Mattar (I994), a escala Stapel é uma modificação da escala de diferencial semântico, diferenciando-se desta última por apresentar um maior número de pontos (io no total) e possuir os pontos representados por números. De forma análoga, Aaker, Kumar e Day (200I) descrevem a escala de Stapel como uma versão simplificada da escala de diferencial semântico, a qual utiliza apenas um pólo em vez de dois.

A vantagem desta escala, segundo Malhotra (200I) e Aaker, Kumar e Day (200I), é que ela não exige um pré-teste dos adjetivos ou frases para assegurar verdadeira bipolaridade. 


\section{ABORDAGEM DE CARVALHO E LEITE}

Um processo alternativo para mensurar a importância de atributos foi proposto por Carvalho e Leite (I999). Segundo esta metodologia, os respondentes eram solicitados a classificar, por ordem decrescente de importância, os seis atributos mais importantes entre os 39 listados no questionário. A justificativa para este procedimento reside na literatura sobre escolha entre produtos, segundo a qual um consumidor avalia apenas um grupo reduzido de marcas - entre duas a oito - em uma determinada compra. Entretanto, para uma situação de pesquisa por meio de questionário de autopreenchimento, os autores sugeriram que seis atributos seriam um valor ideal.

O método ainda previa o cálculo de uma pontuação parcial para cada atributo baseada no número de vezes que o atributo era escolhido em cada uma das seis ordenações de importância, multiplicado pelos seus respectivos pesos, os quais eram assumidos arbitrariamente como sendo: peso seis para o primeiro posto, cinco para o segundo e assim por diante. A importância de cada atributo, então, seria calculada dividindo-se a pontuação parcial de cada um dos atributos pelo somatório das pontuações parciais de todos os atributos. Contudo, a abordagem de Carvalho e Leite (I999) possui um problema crítico. O procedimento de estabelecer um peso predeterminado para os seis postos (seis para o primeiro posto, cinco para o segundo posto, e assim sucessivamente) é arbitrário.

\section{METODOLOGIA}

O problema da pesquisa efetuada foi: como diferentes atributos utilizados para um serviço ofertado podem ser mensurados utilizando-se três escalas diferentes?

As variáveis independentes foram as de caráter demográfico, as quais não informadas em função da solicitação de sigilo por parte dos respondentes e do proprietário do estabelecimento estudado. As variáveis dependentes foram os atributos associados à escolha do serviço oferecido, a seguir apresentadas.

\section{1 DESENVOLVIMENTO DA ABORDAGEM DE PONTOS DECRESCENTES}

O estudo de um evento qualitativo não remete necessariamente à mensuração com variáveis qualitativas. Pereira (200I) ressalta que é totalmente pertinente que o pesquisador considere a utilização de medidas mais elementares (contínuas, discretas), que lhe permitirão maior flexibilidade de análise, já que poderão 
sempre comportar transformações que não seriam possíveis em sentido contrário. Entretanto, o autor lembra que o pesquisador não deve se descuidar de interpretar a representação qualitativa de suas medidas.

Pereira (200I), ao trabalhar com variáveis categóricas, lembra que o pesquisador deve estar consciente da perda de precisão e de examinar se a categorização que faz das manifestações do evento que estuda é a melhor representação do real. Por fim, a escolha do tipo de variável a ser utilizado pelo pesquisador dependerá da natureza de seu objeto de estudo e dos recursos de que disponha para mensuração.

A principal preocupação no desenvolvimento desta nova abordagem está na criação de uma medida quantitativa que represente com fidedignidade as diferenças nos níveis de importância de um dado conjunto de atributos. Dessa forma, a abordagem de pontos decrescentes procura superar alguns aspectos negativos encontrados nas escalas anteriormente citadas (Stapel, diferencial semântico, Likert, escalonamentos do tipo Q, de soma constante, por ordem de posto, por comparação por pares), as quais podem ser citadas:

- como na maioria dos casos existem muitos atributos para serem avaliados, o uso do escalonamento por comparação por pares ou o escalonamento por ordem de posto acaba se tornando cansativo e inviável;

- o escalonamento por ordem de posto gera dados ordinais;

- o uso de mais de cinco atributos gera confusão, dificuldade e fadiga para os respondentes quando o escalonamento de soma constante é utilizado. Além disso, o escalonamento de soma constante não é recomendado quando a população-alvo da pesquisa possui baixa escolaridade;

- o escalonamento tipo Q, apesar de ser uma técnica muito interessante, é de difícil utilização na prática. Além disso, não pode ser utilizada em questionário auto-aplicado;

- as escalas de multiitens, tais como a escala Likert, sofrem a influência dos erros de leniência e da tendência central;

- a escala de diferencial semântico, quando elaborada para medir importância, pode gerar baixa discriminação dos itens e altas curtoses;

- a abordagem de Carvalho e Leite (I999) se baseia em uma ponderação arbitrária.

Para tentar resolver alguns desses problemas, foi proposta a abordagem de pontos decrescentes em dois passos. No primeiro passo, semelhantemente à abordagem proposta por Carvalho e Leite (I999), os respondentes são solicitados a enumerar, por ordem decrescente de importância, seis atributos de uma lista prévia contendo todos os atributos a serem analisados. Este procedimento 
está ancorado no pressuposto da racionalidade limitada dos indivíduos. Como aborda Besanko, Dranove e Shanley (2000), este conceito se refere à capacidade limitada de indivíduos em processar informações, lidar com complexidade e perseguir objetivos racionais. Segundo este conceito, as pessoas não conseguem analisar ou enumerar todo o contingente de informações que surge para elas durante uma transação. Uma forma de lidar com este conceito é partir do pressuposto de que as pessoas, perante um grande número de informações, tendem a limitar sua análise a apenas alguns aspectos considerados principais para a tomada de decisão. Como objetivos deste passo buscou-se encontrar, dentro da gama de atributos total, os atributos considerados como mais importantes (medida ordinal qualitativa). Adicionalmente procurou-se reduzir o tempo necessário para responder ao questionário e, assim, não levar os respondentes à fadiga ou ao aborrecimento.

No segundo passo, os respondentes foram solicitados a atribuir notas de importância, variando de o a Io०, aos atributos escolhidos na primeira etapa, segundo um processo comparativo com o atributo escolhido em primeiro lugar, o qual, por definição, recebe nota Ioo. Depois, as notas atribuídas a cada um dos atributos são somadas, totalizando a pontuação de cada atributo. Por fim, divide-se a pontuação de cada atributo pelo somatório de todos os atributos tomados conjuntamente, resultando no valor de importância de cada atributo. Assim, este segundo passo apresentou os objetivos de incluir uma medida quantitativa das diferenças de postos; facilitar a atribuição de notas, evitando utilizar o escalonamento de soma constante e finalmente incluir um ponto de referência (o atributo escolhido como mais importante), procurando melhorar a discriminação dos itens.

Como resultado do procedimento, foi possível avaliar os dados tanto por uma abordagem qualitativa (primeiro passo) quanto pela abordagem quantitativa (segundo passo). Uma outra vantagem deste procedimento foi que ele procurou detectar pequenas diferenças entre os objetos de estímulo.

\subsection{ORGANIZAÇÃO DA PESQUISA}

O primeiro objetivo desta pesquisa visou comparar os resultados obtidos pela utilização das escalas de diferencial semântico, a abordagem de Carvalho e Leite e a proposta de pontos decrescentes quanto à mensuração dos principais fatores que levam as mulheres a escolherem um salão de beleza. O motivo da escolha deste segmento de serviços se deveu ao fato de que a pesquisa poderia ser realizada rapidamente, como também poderia contar com uma maior participação dos respondentes, visto que eles poderiam preencher o questionário enquanto esperavam para ser atendidos. 
Uma pesquisa exploratória inicial foi realizada para a geração de possíveis atributos para incluir na pesquisa. Esta pesquisa foi, em seguida, depurada por meio de uma entrevista com o proprietário do estabelecimento em que a coleta de dados foi realizada, o qual eliminou alguns itens considerados desnecessários. Como resultado dessa triagem, o questionário final apresentou nove atributos a serem avaliados, os quais são apresentados na Tabela I. É interessante ressaltar que, como o foco desta pesquisa está essencialmente no procedimento metodológico de mensuração da importância dos atributos, o simples fato de algum outro atributo ter sido deixado de lado não deve representar um grande problema.

\section{TABELA I}

ATRIBUTOS CONSIDERADOS NA ESCOLHA DE UM SALAO DE BELEZA

\begin{tabular}{cl}
\hline ATRIBUTO & \multicolumn{1}{c}{ DESCRIÇÃO } \\
\hline F1 & - Confiança na habilidade do profissional (cabeleireiro, pedicure, manicure etc.) \\
F2 & - Conveniência: salão perto do trabalho/casa \\
F3 & - Preço \\
F4 & - Facilidade de estacionamento \\
F5 & - Tempo de espera para começar a ser atendida \\
F6 & - Horário de atendimento \\
F7 & - Produtos utilizados pelo profissional durante a prestação de serviço \\
F8 & - Nível de atendimento (profissional atencioso, salão possui TV, café, água etc.) \\
F9 & - Higiene \\
\hline
\end{tabular}

Fonte: Elaborado pelos autores.

Como segundo objetivo da pesquisa, foram testados dois formatos de apresentação das instruções relacionados à abordagem de pontos decrescentes. No primeiro formato, as instruções estavam divididas em duas partes e dentro de caixas de texto com setas indicativas próximas às escalas (formato I), enquanto no segundo formato todas as instruções necessárias para o preenchimento dos dois passos estavam colocadas juntamente (formato 2).

A amostra foi constituída por 64 mulheres escolhidas ao acaso, por conveniência, de forma não probabilística (ordem de chegada), que completaram uma das quatro versões de questionários (Tabela 2) preparados contendo a escala de diferencial semântico e uma das duas abordagens de pontos decrescentes, invertendo-se a ordem em que as escalas apareciam no questionário. Este procedimento foi incluído na tentativa de eliminar o erro associado normalmente à menor taxa de preenchimento da segunda escala do questionário, como também das possíveis influências que o preenchimento da primeira escala poderia causar no preenchimento da segunda escala. 


\section{TABELA 2}

\begin{tabular}{cll} 
& \multicolumn{1}{c}{ VERSOES DO QUESTIONARIO DE PESQUISA } \\
\hline QUESTIONÁRIO & \multicolumn{1}{c}{ PARTE 1 } & \multicolumn{1}{c}{ PARTE 2 } \\
\hline 1 & Pontos Decrescentes (Formato 1) & Diferencial Semântico \\
2 & Diferencial Semântico & Pontos Decrescentes (Formato 1) \\
3 & Pontos Decrescentes (Formato 2) & Diferencial Semântico \\
4 & Diferencial Semântico & Pontos Decrescentes (Formato 2) \\
\hline
\end{tabular}

Fonte: Elaborado pelos autores.

\section{ANÁlise dos dAdOS}

\subsection{ABORDAGEM DE PONTOS DECRESCENTES}

Dos dois formatos de questionários utilizados para avaliar a abordagem de pontos decrescentes, o formato I apresentou maiores taxas de preenchimento completo $(84,4 \%)$ do que o formato $2(8 \mathrm{I}, 3 \%)$. Além disso, o formato I apresentou também menores erros de preenchimento, isto é, a porcentagem de questionários em que a pontuação atribuída aos fatores seguiu a ordenação decrescente de pontos - essência desta abordagem - foi maior ( $78 \%$ contra $54 \%$ do formato 2).

Da mesma forma que a escala de diferencial semântico e a abordagem de Carvalho e Leite, a abordagem de pontos decrescentes também identificou o fator I como o atributo mais importante ( $\mathrm{I} 8, \mathrm{I} \%)$, seguido pelo fator 9 ( $\mathrm{I} 6,4 \%)$. Os resultados da abordagem de pontos decrescentes são mostrados na Tabela 3.

TABELA 3

IMPORTANCIA DOS ATRIBUTOS SEGUNDO A ABORDAGEM DE PONTOS DECRESCENTES

\begin{tabular}{lccccccccc}
\hline \multicolumn{10}{c}{ ABORDAGEM } \\
& F1 & F2 & F3 & F4 & F5 & F6 & F7 & F8 & F9 \\
\hline Pontos & 3.295 & 1.410 & 2.295 & 1.020 & 2.430 & 1.159 & 2.090 & 1.545 & 2.990 \\
(\%) Pontos & 18,15 & $7,7 \%$ & $12,6 \%$ & $5,6 \%$ & $13,3 \%$ & $6,4 \%$ & $11,5 \%$ & $8,5 \%$ & $16,4 \%$ \\
\hline
\end{tabular}

Fonte: Dados da pesquisa.

\section{2 ABORDAGEM DE CARVALHO E LEITE}

Sob esta abordagem, das 56 pessoas que completaram totalmente a ordenação dos seis atributos requisitados, o fator I foi considerado o atributo mais 
importante, com $24 \%$ dos pontos totais, seguido pelo fator 9 , com $18,9 \%$. O resultado completo da abordagem de Carvalho e Leite é mostrado na Tabela 4.

\section{TABELA 4}

IMPORTANCIA DOS PRINCIPAIS FATORES QUE LEVAM AS MULHERES

A ESCOLHER UM SALÃO DE BELEZA, CONFORME ABORDAGEM DE CARVALHO E LEITE

\begin{tabular}{|c|c|c|c|c|c|c|c|c|}
\hline \multicolumn{9}{|c|}{ ABORDAGEM DE CARVALHO \& LEITE } \\
\hline \multirow{3}{*}{ FATORES } & \multicolumn{6}{|c|}{ NÚMERO DE VEZES EM QUE O FATOR FOI ESCOLHIDO NA: } & \multirow{3}{*}{ PONTOS } & \multirow{3}{*}{ (\%) PONTOS } \\
\hline & $1^{\circ}$ & $2^{\circ}$ & $3^{\circ}$ & $4^{\circ}$ & $5^{\circ}$ & $6^{\circ}$ & & \\
\hline & ESCOLHA & ESCOLHA & ESCOLHA & ESCOLHA & ESCOLHA & ESCOLHA & & \\
\hline F1 & 34 & 10 & 4 & 1 & 3 & 3 & 282 & $24,0 \%$ \\
\hline F2 & 0 & 3 & 3 & 4 & 4 & 8 & 55 & $4,7 \%$ \\
\hline F3 & 2 & 11 & 11 & 9 & 3 & 4 & 148 & $12,6 \%$ \\
\hline F4 & 0 & 1 & 4 & 4 & 6 & 12 & 57 & $4,8 \%$ \\
\hline F5 & 1 & 6 & 7 & 13 & 14 & 5 & 136 & $11,6 \%$ \\
\hline F6 & 0 & 3 & 4 & 9 & 2 & 6 & 68 & $5,8 \%$ \\
\hline F7 & 1 & 6 & 8 & 8 & 11 & 8 & 122 & $10,4 \%$ \\
\hline F8 & 3 & 4 & 5 & 3 & 6 & 7 & 86 & $7,3 \%$ \\
\hline F9 & 15 & 12 & 10 & 5 & 7 & 3 & 222 & $18,9 \%$ \\
\hline
\end{tabular}

Fonte: Dados da pesquisa.

\section{3 ESCALA DE DIFERENCIAL SEMÂNTICO}

Cerca de 58 pessoas preencheram completamente e retornaram o questionário contendo a escala de diferencial semântico. Considerando todas as notas dos atributos analisados, a média de pontos foi de 52,84 de 63 possíveis (nove fatores vezes sete pontos da escala) e desvio padrão de apenas 6,4I, o que representa um coeficiente de variação de apenas i2,13\%. Esses dados mostram que a média dos fatores foi elevada, isto é, a maior parte dos fatores foi considerada importante pela maioria dos respondentes, sendo que poucas notas baixas foram dadas, haja vista o baixo desvio-padrão da amostra.

Em relação à importância dos fatores analisados, o fator I - confiança na habilidade do profissional, com 400 pontos, e o fator 9 - higiene, com 399 pontos, foram os atributos considerados mais importantes. A Tabela 5 mostra os resultados encontrados para a escala de diferencial semântico. 


\section{TABELA 5}

IMPORTANCIA DOS ATRIBUTOS SEGUNDO A ESCALA

DE DIFERENCIAL SEMÂNTICO

\begin{tabular}{lcccccccccc}
\hline \multicolumn{1}{c}{ ESCALA DE DIFERENCIAL SEMÂNTICO } & & & \\
& & F1 & F2 & F3 & F4 & F5 & F6 & F7 & F8 & F9 \\
\hline Pontos & & & & & & & & & \\
Formato 1 & 216 & 142 & 176 & 150 & 202 & 186 & 190 & 175 & 213 \\
Formato 2 & 184 & 101 & 160 & 135 & 177 & 155 & 166 & 151 & 186 \\
Total & 400 & 243 & 336 & 285 & 379 & 341 & 356 & 326 & 399 \\
Média de pontos & & & & & & & & & \\
Formato 1 & 6,97 & 4,58 & 5,68 & 4,84 & 6,52 & 6,00 & 6,13 & 5,65 & 6,87 \\
Formato 2 & 6,81 & 3,74 & 5,93 & 5,00 & 6,56 & 5,74 & 6,15 & 5,59 & 6,89 \\
Total & 6,90 & 4,19 & 5,79 & 4,91 & 6,53 & 5,88 & 6,14 & 5,62 & 6,88 \\
Importância & & & & & & & & & \\
Formato 1 & $13,1 \%$ & $8,6 \%$ & $10,7 \%$ & $9,1 \%$ & $12,2 \%$ & $11,3 \%$ & $11,5 \%$ & $10,6 \%$ & $12,9 \%$ \\
Formato 2 & $13,0 \%$ & $7,1 \%$ & $11,3 \%$ & $9,5 \%$ & $12,5 \%$ & $11,0 \%$ & $11,7 \%$ & $10,7 \%$ & $13,2 \%$ \\
Total & $13,1 \%$ & $7,9 \%$ & $11,0 \%$ & $9,3 \%$ & $12,4 \%$ & $11,1 \%$ & $11,6 \%$ & $10,6 \%$ & $13,0 \%$ \\
\hline
\end{tabular}

Fonte: Dados da pesquisa.

\section{CONSIDERAÇÕES FINAIS}

Antes de analisar os resultados conjuntamente, é interessante destacar as dificuldades da comparação das abordagens entre si. Embora tenham sido utilizadas para medir o mesmo objeto - a importância de atributos na escolha de um salão de beleza -, as abordagens possuem características peculiares, como a natureza dos dados e a forma de construção, o que, na prática, resulta na dificuldade de comparação por métodos estatísticos mais rigorosos.

A Tabela 6 apresenta resumidamente os dados finais de cada uma das abordagens analisadas. Uma primeira comparação entre essas abordagens pode ser realizada verificando-se o coeficiente de correlação de Pearson (Tabela 7), calculado com base nos dados métricos de importância resultantes em cada abordagem. Segundo esses resultados, as três abordagens apresentam alta correlação entre si, o que apresenta sinais de que as escalas mediram o mesmo objeto e, de certa forma, chegaram a um resultado bastante próximo entre elas. Em destaque, é citada a alta correlação entre as escalas de Carvalho e Leite e a abordagem de pontos decrescentes. De forma análoga, a comparação de postos medida pelo rho de Spearman também apresentou alta similaridade entre as abordagens consideradas, indicando consistência dos resultados. 


\section{TABELA 6}

RESUMO DOS DADOS FINAIS

\section{DAS ABORDAGENS UTILIZADAS}

\begin{tabular}{ccccccc}
\hline ATRIBUTO & \multicolumn{3}{c}{ IMPORTÂNCIA } & \multicolumn{3}{c}{ IMPORTÂNCIA } \\
& DS & C\&L & PD & DS & C\&L & PD \\
\hline F1 & $13,1 \%$ & $24,0 \%$ & $18,07 \%$ & 1 & 1 & 1 \\
F2 & $7,9 \%$ & $4,7 \%$ & $7,73 \%$ & 9 & 9 & 7 \\
F3 & $11,0 \%$ & $12,6 \%$ & $12,59 \%$ & 6 & 3 & 4 \\
F4 & $9,3 \%$ & $4,8 \%$ & $5,59 \%$ & 8 & 8 & 9 \\
F5 & $12,4 \%$ & $11,6 \%$ & $13,33 \%$ & 3 & 4 & 3 \\
F6 & $11,1 \%$ & $5,8 \%$ & $6,36 \%$ & 5 & 7 & 8 \\
F7 & $11,6 \%$ & $10,4 \%$ & $11,46 \%$ & 4 & 5 & 5 \\
F8 & $10,6 \%$ & $7,3 \%$ & $8,47 \%$ & 7 & 6 & 6 \\
F9 & $13,0 \%$ & $18,9 \%$ & $16,40 \%$ & 2 & 2 & 2 \\
\hline
\end{tabular}

Legenda: (DS) Abordagem de Diferencial Semântico; (C\&L) Abordagem de Carvalho e Leite; (PD) Abordagem de Pontos Decrescentes.

Fonte: Dados da pesquisa.

\section{TABELA 7}

\begin{tabular}{|c|c|c|c|c|}
\hline \multicolumn{5}{|c|}{$\begin{array}{l}\text { CORREAÇAO ENTRE } \\
\text { AS ABORDAGENS }\end{array}$} \\
\hline & & DS & $C \& L$ & GHIS \\
\hline \multirow[t]{3}{*}{ DS } & Correlação de Pearson & 1,000 & $0,816^{* *}$ & $0,810^{* *}$ \\
\hline & Sig. (2-tailed) & & 0,007 & 0,008 \\
\hline & $\mathrm{N}$ & 9 & 9 & 9 \\
\hline \multirow[t]{3}{*}{$C \& L$} & Correlação de Pearson & $0,816^{* *}$ & 1,000 & $0,964^{* *}$ \\
\hline & Sig. (2-tailed) & 0,007 & . & 0,000 \\
\hline & $\mathrm{N}$ & 9 & 9 & 9 \\
\hline \multirow[t]{3}{*}{ PD } & Correlação de Pearson & $0,810^{* *}$ & 0,964 & 1,000 \\
\hline & Sig. (2-tailed) & 0,008 & 0,000 & . \\
\hline & $\mathrm{N}$ & 9 & 9 & 9 \\
\hline
\end{tabular}

*** A correlaçnao é significativa no o,or nível (2-tailed)

Fonte: Dados da pesquisa. 


\section{TABELA 8}

\section{CORRELACAO ENTRE AS ABORDAGENS}

\begin{tabular}{|c|c|c|c|c|c|}
\hline & & & DS & $C \& L$ & PD \\
\hline \multirow[t]{9}{*}{ Spearman rho } & DS & Coeficiente de Correlação & 1,000 & $0,867^{* *}$ & $0,833^{* *}$ \\
\hline & (ordinal) & Sig. (2-tailed) & . & 0,002 & 0,005 \\
\hline & & $\mathrm{N}$ & 9 & 9 & 9 \\
\hline & C\&L & Coeficiente de Correlação & $0,867^{\star *}$ & 1,000 & $0,933^{* *}$ \\
\hline & (ordinal) & Sig. (2-tailed) & 0,002 & . & 0,000 \\
\hline & & $\mathrm{N}$ & 9 & 9 & 9 \\
\hline & PD & Coeficiente de Correlação & $0,833^{* *}$ & $0,933^{* *}$ & 1,000 \\
\hline & (ordinal) & Sig. (2-tailed) & 0,005 & 0,000 & . \\
\hline & & $\mathrm{N}$ & 9 & 9 & 9 \\
\hline
\end{tabular}

** A correlação é significativa no o,or nível (2-tailed)

Fonte: Dados da pesquisa.

Considerando os dados métricos de importância, foi possível perceber ainda grandes diferenças nos valores dos atributos entre as abordagens. A maior diferença foi verificada na escala de diferencial semântico. Devido ao fato de que a escala de diferencial semântico apresentou altas médias para a maioria dos atributos e baixo desvio-padrão geral, a diferença entre o atributo mais importante $(\mathrm{I} 3, \mathrm{I} \%)$ e o menos importante $(7,9 \%)$ foi pequena $(5, \mathrm{I} \%)$, comparada com a abordagem de Carvalho e Leite (I9,3\%) e a abordagem de pontos decrescentes $(\mathrm{I} 2,5 \%)$. Ainda por esta abordagem, vários atributos foram considerados importantes, visto que a diferença entre muitos atributos foi pequena.

Esses resultados sugerem que a escala de diferencial semântico não consegue discriminar bem os atributos, visto que os respondentes acabam por considerar todos os atributos importantes e, por sua vez, a escala de Carvalho e Leite foi a que melhor conseguiu discriminar os atributos.

Entretanto, uma questão que fica implícita nesse contexto é se todos, ou melhor, se muitos dos atributos foram mesmo tão importantes como a escala de diferencial semântico está sugerindo. Como os valores reais do objeto em análise não eram conhecidos, foram utilizados meios alternativos para responder a esta questão. Os mesmos respondentes que preencheram a escala de diferencial semântico também preencheram as escalas de Carvalho e Leite e a de pontos decrescentes. Se realmente muitos dos atributos fossem importantes, como sugeria a escala de diferencial semântico, seria plausível esperar que esses atributos obtivessem uma participação razoável quando da escolha do atributo mais importante na escala de Carvalho e Leite. Entretanto, o atributo I concentrou 60\% dessas escolhas e, ao ser considerado em conjunto com o atributo 9 tem-se um total de $87 \%$ das escolhas. 
Esses resultados reforçaram a idéia de que os respondentes possuíam uma predisposição para atribuir notas elevadas para a maioria dos atributos. Quando forçados a realizar uma escolha ordenada de importância, as diferenças entre os atributos acabaram emergindo. A questão então foi como quantificar essas diferenças.

Nesse sentido, as abordagens de Carvalho e Leite e de pontos decrescentes apresentaram-se como opções. Entretanto, a abordagem de pontos decrescentes apresentou duas falhas quanto à sua proposta inicial. Mostrando a mesma deficiência que a escala de diferencial semântico, a abordagem de pontos decrescentes não conseguiu êxito na discriminação dos itens em análise, visto a alta média $(86,83)$ e desvio-padrão $(\mathrm{I} 2,34)$ atribuídos aos seis itens escolhidos previamente pelos respondentes. Pela mesma razão, o objetivo de detectar pequenas diferenças entre os objetos de estímulo também não foi alcançado. Um outro problema da abordagem de pontos decrescentes que ainda mereceu destaque foi a taxa de erro de preenchimento da escala ( $22 \%$ dos questionários do formato I e $46 \%$ dos questionários do formato 2), o que demonstrou uma grande dificuldade de entendimento das instruções de preenchimento de ambos os formatos. Por esta razão, pesquisas futuras poderiam avaliar o resultado de novos formatos de instrução, bem como a utilização da abordagem em forma de entrevista pessoal com cartões em vez de questionário.

Quanto à escala de Carvalho e Leite, esta se mostrou mais realista aos resultados apresentados anteriormente. Entretanto, dada a arbitrariedade com que são estipulados os pesos das escolhas, pesquisas futuras poderiam analisar o impacto da utilização dessa abordagem em diferentes situações de pesquisa, como, por exemplo, a extensão de atributos utilizados e a complexidade do processo de tomada de decisão (solução de problema ampliada ou solução de problema limitada), entre outros.

Como uma sugestão à abordagem de Carvalho e Leite, recomenda-se a apresentação da importância dos atributos em uma escala de ০\% a ı००\%, dividindo-se o valor obtido por cada atributo pelo número 28,57\% (6/2I), que é a pontuação máxima que um atributo pode atingir (situação em que ı००\% dos respondentes escolhem um determinado atributo como o mais importante, mantidos os pesos e o número de escolhas conforme sugerido pelos autores). Dessa forma, os dados representariam o percentual atingido com o máximo que poderia ser conseguido.

\section{REFERÊENCIAS}

AAKER, D. A.; KUMAR, V.; DAY, G. S. Pesquisa de marketing. São Paulo: Atlas, 200 I. BESANKO, D.; DRANOVE, D.; SHANLEY, M. Economics of strategy. New York: John Wiley \& Sons, Inc., 2000. 
BROWN, T. J.; CHURCHILL, G. A.; PETER, J. P. Improving the measurement of service quality. Journal of Retailing, v. 69, n. I, p. I27-I39, I993.

CARMAN, J. M. Consumer perceptions of service quality: an assessment of the servqual dimensions. Journal of Retailing, v. 66, p. 33-55, Spring, I990.

CARVALHO, F. A. de; LEITE, V. F. Atribute importance in service quality: an empirical test of the PBZ conjecture in Brazil. International Journal of Service Industry Management, v. IO, n. 5, p. 487-504, I999.

CHURCHILL, G. A. Jr. Marketing research: methodological foundations. 5. ed. Orlando: The Dryden Press, I99I.

COOPER, D. R.; SCHINDLER, P. S. Métodos de pesquisa em administração. 7. ed. Porto Alegre: Bookman, 2003.

CRONIN, J. J.; TAYLOR, S. A. Measuring service quality: a reexamination and extension. Journal of Marketing, v. 56, p. 55-68, July I992.

- Servperf versus Servqual: reconciling performance-based and perceptionsminus-expectations measurement of service quality. Journal of Marketing, v. 58, p. I253I, Jan. I994.

ENGEL, J. F.; BLACKWELL, R. D.; MINIARD, P. W. Comportamento do Consumidor. 8. ed. Rio de Janeiro: LTC, 2000.

GIANESI, I. N.; CORRÊA, H. L. Administração estratégica de serviços: operações para a satisfação do cliente. São Paulo: Atlas, I994.

KINNEAR, T. C.; TAYLOR, J. R. Marketing research: an applied approach. $5^{\text {th }}$ ed. New York: McGraw-Hill Inc., I996.

KOTLER, P.; ARMSTRONG, G. Princípios de marketing. 7 ed. Rio de Janeiro: LTC, I999. LOVELOCK, C.; WRIGHT, L. Serviços: marketing e gestão. São Paulo: Saraiva, 200I. 4I6 p. MALHOTRA, N. K. Pesquisa de marketing: uma orientação aplicada. 3. ed. Porto Alegre: Bookman, 200I

MATTAR, F. N. Pesquisa de marketing. 2. ed. São Paulo: Atlas, I994. v. I.

PARASURAMAN, A.; BERRY, L. L.; ZEITHAML, V. A. A conceptual model of service quality and its implication for future research. Journal of Marketing, v. 49, p. 4I-5O, Fall, I985.

- Reassessment of expectations as a comparison standard in measuring service quality: implication for further research. Journal of Marketing, v. 58, p. III-24, jan., I994. - Refinement and reassessment of the servqual scales. Journal of Retailing, v. 67, n. 4 , p. 420-450, I99I.

- Servqual: a multiple-item scale for measuring consumer perceptions of service quality. Journal of Retailing, v. 64, n. I, p. I2-40, I988.

PASQUALI, L. Psicometria: teoria e aplicações. Brasília: UNB, I997.

PEREIRA, J. C. R. Análise de dados qualitativos: estratégias metodológicas para as ciências da saúde, humanas e sociais. São Paulo: Edusp, 200I.

PINDYCK R.; RUBINFELD D. L. Microeconomia. 5. ed. São Paulo: Prentice Hall, 2002. QUESTER, P.; WILKINSON, J. W.; ROMANIUK, S. A test of four service quality measurement scales: the case of the Australian advertising industry. Working paper 39, Cen- 
tre de Recherche et d'Etudes Appliquées, Group esc Nantes Atlantique, Graduate School of Management, Nantes, France, I995.

ROBLEDO, M. A. Measuring and managing service quality: integrating customer expectations. Managing Service Quality, v. II, n. I, p. 22-3I, 200 I.

TAYLOR, S. A. Service quality and consumer attitudes: reconciling theory and measurement. In: SWARTZ, T. A.; BOWEN, D. E.; BROWN, S. W. (Eds.). Advances in services marketing and management. Greenwich, CT: JAI Press, I995. v. 4, p. I-36.

TEAS, R. K. Expectations, performance evaluation, and consumer's perceptions of quality. Journal of Marketing, v. 57, n. 4, p. I8-34, I993.

WEINSTEIN, A. Segmentação de mercado. São Paulo: Atlas, I995.

WIRTZ, J. Improving the measurement of customer satisfaction: a test of three methods to reduce halo. Managing Service Quality, v. II, n. 2, p. 99-III, 200 I.

ZEITHAML, V.; BITNER, M. Marketing de serviços: a empresa com foco no cliente. Porto Alegre: Bookman, 2003.

\section{TRA M I TAÇ Ã 0}

Recebido em 16/9/2005

Aprovado em 26/5/2006 
Copyright of Revista de Administração Mackenzie is the property of Universidade Presbiteriana Mackenzie, RAM-Revista de Administracao Mackenzie and its content may not be copied or emailed to multiple sites or posted to a listserv without the copyright holder's express written permission. However, users may print, download, or email articles for individual use. 